\title{
CONTENIDO GENERADO POR LOS USUARIOS, SU RELACIÓN CON LA PERSONALIDAD DE MARCA Y EL VALOR DE MARCA
}

USER GENERATED CONTENT, ITS RELATIONSHIP WITH BRAND PERSONALITY AND BRAND EQUITY

\author{
Mario Antonio Ruiz-Aguilar \\ Universidad Autónoma de Guadalajara (México) \\ mario.ruiz@edu.uag.mx \\ orcid.org/0000-0002-8733-1771 \\ Rafael Avalos-Pelayo \\ Universidad Autónoma de Guadalajara (México) \\ ravalos@edu.uag.mx \\ orcid.org/0000-0002-0595-1986
}

Forma parte de una investigación realizada para la obtención del grado de Doctor en Administración por la Universidad Autónoma de Guadalajara marca y el valor de marca. index.comunicación, 10(1), 125-147. 
Resumen: Las redes sociales digitales (RSD) son una gran herramienta de comunicación de marca para las organizaciones en la actualidad. Debido a que las investigaciones en México son escasas, es relevante conocer cómo los contenidos que los consumidores crean en estas plataformas se relacionan con las percepciones que ellos desarrollan acerca de las marcas. Se realizó una investigación cuantitativa que tuvo como objetivo determinar las relaciones entre tres variables perceptuales, las cuales son los contenidos generados por los usuarios (CGU), la personalidad de marca (PM) y el valor de marca con base en el consumidor (VMBC) referente al sector teléfonos móviles inteligentes (smartphones). Con una muestra de 211 usuarios de teléfonos celulares se hizo un análisis factorial confirmatorio, a partir del cual se elaboró un modelo de ecuaciones estructurales, con el que se encontró que la PM influye a los CGU y el VMBC, pero que los CGU no influyen directamente en el VMBC en el contexto mexicano de la Zona Metropolitana de Guadalajara.

Palabras clave: valor de marca; personalidad de marca; contenido social; consumidor; redes sociales; ecuaciones estructurales.

Abstract: Social Networks are nowadays a very important way for companies to make brand communication. Because studies about this topic in Mexico are scarce it is relevant to know how User Generated Content that consumers communicate through these social platforms is related with the perception they have about brands. A quantitative study was done as part of a doctoral thesis, which had as main objective to determine the relationship there is between these three variables: User Generated Content (UGC), Brand Personality (BP), and Consumer Based Brand Equity (CBBE). Using a sample of 211 smartphone users, a Confirmatory Factor Analysis was conducted from which a Structural Equation Model was developed, finding that BP has an impact on UGC and CBBE, but UGC are not related directly to CBBE in the Mexican context of the metropolitan area of Guadalajara.

Keywords: Brand Equity; Brand Personality; User Generated Content; Consumer; Social Networks; Structural Equation System. 


\section{Introducción}

Las redes sociales digitales (RSD o redes sociales) forman parte de la llamada Web 2.0, fase del desarrollo del internet que permite alta interacción, conversaciones entre individuos y empresas, creación de comunidades e involucramiento de usuarios en la generación de contenido (Sashi, 2012). Las principales redes sociales suman entre todas 4.200 millones de usuarios (Data Reportal, 2019; Socialbakers, s.f.), de los cuales México cuenta con 88 millones, dato que significa una penetración del $67 \%$ de la población. En total, las comunicaciones online en redes sociales de todo el mundo alcanzaron un valor de US\$33 billones y la publicidad online US\$84 billones (Page, Firth, y Rand, 2016). El 89\% de los mexicanos utilizan el internet para visitar RSD y un 39\% hacen compras en línea, mientras que $40 \%$ las hacen tanto en formato tradicional como digital (Asociación de Internet, 2018).

La utilización de contenidos en línea es cada vez más común entre las organizaciones y ha sustituido a la publicidad tradicional (offline) debido a los bajos costos y alcance. Las redes sociales permiten a las organizaciones sostener relaciones directas con los consumidores en una comunicación multidireccional, mediante la cual la empresa recaba información de ellos y estos con otras personas que, de manera similar, retroalimentan a la organización. Las organizaciones no son las únicas que comunican en redes sociales, ya que los usuarios también generan contenidos acerca de las marcas, comparten información y socializan respecto a ellas. Por esto, las marcas han dejado de estar bajo el total control de las organizaciones, cediéndole gran parte del mando a los consumidores (Schivinski y Dabrowski, 2016).

La marca es uno de los activos intangibles más importantes de una organización y la percepción que los consumidores tengan de ella se ve reflejado en los resultados económicos que provocan. Según la Asociación de Internet (2018), uno de cada tres mexicanos sigue interactuando con la publicidad que ven en línea y el $21 \%$ ha realizado una compra después de haber visto comunicación online de determinada marca. Esto demuestra la relevancia que tiene comprender la relación entre las comunicaciones en RSD con el desempeño de las marcas.

Gensler, Volckner y Liu-Thompkins (2013) declaran que existe una falta de conocimiento sobre cómo las comunicaciones en medios sociales afectan al desempeño de una marca. Para aminorar este vacío, los investigadores deben centrarse en entender cómo las marcas son procesadas de forma perceptual por los consumidores, ya que de ello dependen los resultados económicos que provoquen en las organizaciones e industrias. 
Entre tales percepciones se encuentra la personalidad de marca (PM), la cual tiene influencia en los contenidos que los usuarios comparten en las redes sociales, algo que es conocido como contenidos generados por los usuarios (CGU). Otro fenómeno relacionado con las marcas es el valor de marca con base en el consumidor (VMBC o valor de marca) el cual también ha sido asociado con la influencia de la personalidad de marca, así como con los contenidos generados por los usuarios (Dickinger y Lalicic, 2015; Loureiro, Lopes y Kaufmann, 2014; Walsh, Clavio, Lovell y Blaszka, 2013).

El objetivo del presente texto es explicar la relación existente entre la personalidad de marca, los CGU y el valor de marca. En correspondencia con dicho objetivo, se ha planteado la siguiente pregunta de investigación: ¿cuál es la relación que existe entre la personalidad de marca, los contenidos generados por los usuarios y el valor de marca en el contexto mexicano?

\section{Marco teórico}

Los contenidos generados por los usuarios (CGU) son definidos por la Organización para la Cooperación y el Desarrollo Económico (OCDE) como aquellos creados por los consumidores o usuarios en medios sociales, cuyas características son las siguientes: son compartidos públicamente en internet, implican algún esfuerzo para crear la publicación o adaptar la de terceros y, en tercer lugar, son creados como actividad no profesional (Vickery y WunschVincent, 2007). En ocasiones, el fenómeno de boca a boca electrónico (EWOM) es usado como sinónimo de CGU y viceversa, no obstante, el EWOM es una de las formas del CGU, pues los últimos son un concepto más amplio que abarca imágenes, animaciones, audio u otros formatos que suponen algún esfuerzo mayor al escrito (Smith, Fischer y Yongjian, 2012).

Existen dos tipos de usuarios: los productores de CGU y quienes son consumidores de estos (Yoo y Gretzel, 2011). Los primeros son aquellos que mediante imágenes, videos, audios o cualquier otro formato exponen a las organizaciones y sus marcas de manera pública en internet, provocando que otros hagan comentarios, evaluaciones y críticas sobre ellas, presionándolas a adecuarse a lo que necesitan y demandan, ya que estas comunicaciones impactan en el comportamiento de otros consumidores (Dellarocas, 2003).

Los CGU pueden darse de tipo experiencial y promocional. El primer tipo se refiere al desempeño de la marca, el cual impulsa al usuario a hacer publicaciones relacionadas a ésta. Los segundos son aquellos que transmiten los beneficios que una marca brinda. Por lo general, los de tipo experiencial gozan de más credibilidad por parte de otros usuarios, más aún cuando son transmitidos de manera orgánica; en otras palabras, sin que la organización 
haya pagado para su difusión, sino que surge y se difunde como parte de la dinámica social de los usuarios en las RSD (Kim y Song, 2017).

El valor de marca con base en el consumidor según Aaker, D. A. (1991) es un constructo que se refiere a la percepción y evaluación subjetiva que el consumidor hace acerca de una marca, los beneficios y los valores que obtiene de ésta. Las dimensiones que componen esta variable son lealtad de marca, notoriedad de marca, asociaciones de marca, calidad percibida, así como otros aspectos organizacionales como registros de marcas y las relaciones con el canal (Aaker D. A., 1991).

La lealtad de marca es la preferencia de una marca en particular sobre las demás disponibles y que lleva al consumidor a tener disposición de recompra. La notoriedad de marca es el grado en que la marca es reconocida y recordada, lo que va de la mano con las asociaciones de marca, las cuales son interpretaciones basadas en recuerdos que el consumidor tiene sobre la marca como producto de las acciones de marketing de la organización. La calidad percibida es una valoración subjetiva acerca del desempeño de la marca (Aaker D. A., 1991).

El valor de marca es un fenómeno perceptual en la mente de los consumidores y se da tanto en ambientes «analógicos» como online (Schivinski y Dabrowski, 2016). Las redes sociales constituyen espacios en internet donde los consumidores tienen más participación que las organizaciones publicando contenidos, mismos que influye a sus pares. De esta forma, las dimensiones del VMBC que Aaker postuló hace tres décadas (Aaker D. A., 1991) están relacionadas directa y positivamente entre sí en los ambientes de redes sociales, de forma similar como sucede en el mundo fuera de internet (Sasmita y Mohd Suki, 2015).

La personalidad de marca se refiere a las características o rasgos humanos de personalidad que los consumidores le asignan a las marcas al interactuar con ellas (Aaker J. L., 1997; Escobar-Farfán, Sánchez y ArayaCastillo, 2016) y está determinada por cinco dimensiones en las que se organizan 42 rasgos de personalidad que están basados en «Los Cinco Grandes» propuestos por Goldberg $(1990 ; 1.218)$. Estas dimensiones son emoción, competencia, sinceridad, sofisticación y rudeza. Aunque el modelo ha recibido críticas por su adaptabilidad a culturas distintas a la estadounidense, sigue siendo el más difundido y aceptado en el ámbito de la investigación (Geuens, Weijters y De Wulf, 2009).

La tendencia a antropomorfizar las marcas es lo que permite que los consumidores desarrollen relaciones con éstas, pues la marca, contrario a lo 
que se pueda percibir, es en realidad un ente activo con el cual el ser humano se relaciona. Los comportamientos de las marcas son productos de las decisiones y acciones de marketing que las organizaciones realizan y estos comportamientos son interpretados por los consumidores como parte de su personalidad, lo que influye en su relación con éstas (Fournier, 1998).

La personalidad de marca también influencia la imagen y personalidad que el consumidor adjudica a la empresa productora o comercializadora del producto, si es que este la identifica. Este fenómeno se hace más presente cuando el nombre de la organización o marca corporativa es el mismo que la marca del producto, por ejemplo, marcas como Huawei, Nike o Adidas (Anderson, Raju y Kordrostami, 2018).

Así, los consumidores eligen a las marcas de la misma manera en que eligen a las personas a su alrededor, como los amigos; en otras palabras, humanizan a las marcas adjudicándoles rasgos similares a los de personas. En este sentido, cuanto más perciba el consumidor concordancia entre su propia personalidad con la personalidad de la marca, aumentarán las probabilidades de que esta sea valorada y adquirida (Yun, Pamuksuz y Duff, 2019 y ToldosRomero y Orozco-Gómez, 2015).

La personalidad de marca cobra aún más importancia en los casos en que la decisión de compra del consumidor es mayormente influenciada por aspectos emocionales como en el caso de los productos de alto involucramiento, los cuales suelen ser percibidos como más complejos, tales como automóviles, computadoras, entre otros (González Hernández, Orozco Gomez y Barrios, 2011). De forma similar sucede en situaciones en que los atributos palpables de las marcas son muy similares entre sí, como los materiales del producto, el precio o el lugar de venta, ya que los consumidores se abocan a designar más peso a los rasgos perceptuales relacionados a la personalidad de las marcas (Akin, 2011).

\subsection{La personalidad de marca y su relación con los contenidos generados por los usuarios y el valor de marca}

La personalidad de marca (PM) influye sobre los contenidos generados por los usuarios (CGU) ya que los consumidores, al percibir los rasgos de personalidad que definen a una marca, generan comunicaciones a través de redes sociales $y$, además, tienden a participar en grupos y comunicar acerca de aquellas marcas que consideran coincidentes con su personalidad (Paschen, Pitt, Kietzmann y Farshid, 2017; Yun et al., 2019).

La percepción de rasgos humanos en una marca es más evidente en quienes son fanes en redes sociales, en comparación con quienes no lo son 
(Walsh et al., 2013). Además, cuantas más experiencias positivas tienen los consumidores con la marca, la personalidad de esta es percibida con mayor intensidad, lo que le da estímulos para compartir contenidos positivos en redes sociales (Kim, Han y Park, 2001). De esa manera las percepciones de rasgos humanos que tienen acerca de la marca se hacen expresas en los contenidos que difunden en las redes sociales (Dickinger y Lalicic, 2015; Yun et al., 2019). Con base en lo anterior, se propone la primera hipótesis:

- H1: En el ambiente de las redes sociales, cuanto más fuerte es la PM, mayores son los contenidos generados por los usuarios.

La personalidad de marca tiene notable influencia directa sobre el valor de marca (Loureiro et al., 2014; Su y Tong, 2016) y se ha comprobado que impacta en cada una de las dimensiones que lo conforman (Chen y Phou, 2013; Su y Tong, 2015). Esto significa que cuando el consumidor percibe la marca como una persona con características meramente humanas, tiende a adjudicarle una mayor valía a la misma. Además, la creación de valor es más fuerte en personas que tienen una percepción simbólica o abstracta de la marca en comparación con aquellos que la interpretan como un objeto meramente tangible (Aggarwal y McGill, 2012; Valette-Florence, Guizani y Merunka, 2011). Asimismo, la PM al impactar en el valor de marca influye a la intención de adquisición por parte de los consumidores (Vahdati y Mousavi Nejad, 2016), es decir, cuanto más valor adjudican a la marca — producto de la percepción de rasgos humanos en ella- mayores serán las probabilidades de que se realice la compra. Con base en esto se propone la hipótesis siguiente:

- H2: En el ambiente de redes sociales, la PM tiene influencia directa sobre el valor de marca con base en el consumidor.

Los contenidos que los usuarios generan en redes sociales tienen relación directa con el valor de marca y con cada una de las dimensiones que lo componen, de manera que acrecientan lo conocida que es la marca y la forma en que es asociada en la mente de los consumidores, lo que a su vez incrementa la calidad que se percibe y la lealtad hacia la misma (Schivinski y Dabrowski, 2015; Schivinski y Dabrowski, 2016). Esta relación también se manifiesta en el ámbito de medios tradicionales, los cuales aumentan la notoriedad de la marca, mientras que en el ámbito de las redes sociales, fomentan la imagen de marca (Bruhn, Schoenmueller y Schafer, 2012).

Lo que los usuarios comunican tiene más impacto en comparación con los contenidos que las empresas transmiten, ya que los consumidores consideran más creíble, auténtico y desinteresado aquello que otra persona le 
dice, en comparación con lo que difunden las organizaciones (Schivinski y Dabrowski, 2016; Scholz, Dorner, Landherr y Probst, 2013). No obstante, si la comunicación creada por las compañías es de naturaleza social y no comercial, provoca mayor conexión emocional con las marcas y con otros usuarios, lo que fomenta la generación de contenidos por parte de consumidores (Ding, Phang, Lu, Chuan-Hoo y Sutanto, 2014). Además, cuando estas comunican más frecuentemente contenidos acerca de las marcas, los usuarios son más impulsados a publicar contenidos propios (Scholz et al., 2013). En contraste, si los CGU son negativos, diluyen el valor de marca, algo que gana más fuerza cuando el consumidor lee las opiniones de sus pares y estas concuerdan con las propias (Bambauer-Sachse y Mangold, 2011; Xia y Bechwati, 2010).

Por último, los consumidores desarrollan un involucramiento o acercamiento emocional con las marcas en redes sociales, lo cual se hace visible a través de lo que ellos comunican y, cuanto mayor sea el valor que perciben, más fuerte es el sentido de comunidad, por lo que se sienten inclinados a generar comentarios acerca de una marca cuando perciben valor y apego a la misma (Christodoulides, Jevons y Bonhomme, 2012). Con lo anterior se puede proponer la tercera hipótesis de investigación:

- H3: En el ambiente de las redes sociales, los contenidos generados por los usuarios tienen relación directa con el valor de marca con base en el consumidor

El modelo de investigación es el de la figura 1, en el cual se aprecian las hipótesis de trabajo.

Figura 1. Modelo teórico de investigación

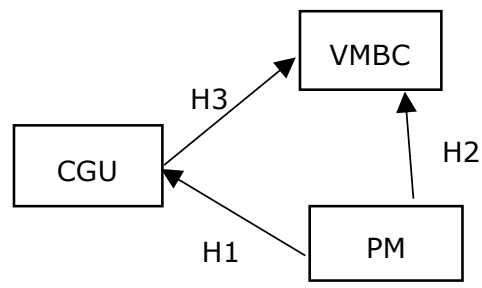

Fuente: elaboración propia

\section{Metodología}

El presente artículo es el resultado de un estudio de corte cuantitativo, tipo cuasi experimental, alcance correlacional explicativo, de temporalidad transversal y nivel confirmatorio. Para lograr el objetivo, se midieron las variables valor de marca con base en el consumidor (VMBC), contenidos 
generados por los usuarios (CGU) y personalidad de marca (PM) mediante un instrumento aplicado con la técnica de encuesta, compuesto por 62 preguntas con respuesta en escala de Likert de cinco puntos cuyos valores fueron: 1: muy de acuerdo, 2: ligeramente de acuerdo, 3: ni de acuerdo ni en desacuerdo, 4: ligeramente en desacuerdo, 5: muy en desacuerdo.

Los 42 ítems de la variable personalidad de marca son los propuestos por Aaker, J.L. (1997) los cuales se dispusieron de esta manera para cada dimensión: 11 para sinceridad, 11 de emocionalidad, 9 de competencia, 6 sofisticación y 5 de rudeza. Por su parte, la variable contenidos generados por los usuarios se midió a través de cuatro ítems propuestos por Kudeshia y Kumar (2017); mientras que la variable VMBC se midió con 16 ítems que se distribuyen según las dimensiones que componen a la variable, así: cuatro ítems de Schivinski y Dabrowski (2016) y cinco de Sasmita y Mohd Suki (2015) midieron la dimensión Notoriedad/Asociaciones de Marca; cuatro ítems de Buil, Martinez y De Chernatony (2013) se usaron para Calidad Percibida y tres ítems de Buil et al. (2013) se utilizaron para medir la Lealtad de Marca.

Los resultados han sido obtenidos a partir un análisis factorial confirmatorio de Modelos de Ecuaciones Estructurales (SEM). El análisis factorial consiste en un grupo de métodos estadísticos multivariantes que buscan explicar la estructura de una matriz de datos de la manera más resumida posible sin que se pierda información (Hair, Anderson, Tatham y Black, 1999).

El análisis factorial confirmatorio se centra en estudiar las estructuras de las variables y la relación que tienen entre ellas en un modelo (Hair Jr., Black, Babin y Anderson, 2014), siendo los SEM la técnica más actualizada y que consiste en una serie de ecuaciones que incorporan regresiones múltiples permitiendo incorporar al estudio variables no observadas, en otras palabras, que no han sido medidas directamente (Hair, Anderson, Tatham y Black, 1999).

Se obtuvo la información acerca de la percepción de los consumidores de teléfonos celulares inteligentes smartphones en la zona metropolitana de Guadalajara, México. Para que los usuarios tuvieran un incentivo de completar el instrumento, se les ofreció que al concluirlo ingresarían al sorteo de un premio de interés para ellos, obteniendo así 254 instrumentos completados.

\subsection{Población, muestra y validez de los datos}

La población se definió como usuarios de smartphones que hacen uso de redes sociales y que han estado expuestos a comunicación respecto de marcas de smartphones. Según datos de la Asociación de Internet (2018), los usuarios de 
smartphones en la zona metropolitana de Guadalajara son 2.223 .033 por lo que para efectos de cálculo, la población es considerada indeterminada (Badii, Castillo y Guillen, 2017).

Para determinar el tamaño de muestra se consideró $\mathrm{P}=0,839$ y $\mathrm{Q}=0,161$, siendo $\mathrm{P}$ la probabilidad compra y $\mathrm{Q}$ la probabilidad de no compra de la marca. Esto fue resultado de la opinión directa del entrevistado, mediante la consulta pretendo comprar nuevamente esta marca en el futuro. El margen de confianza fue de $95 \%$ y el margen de error de $5 \%$. El tamaño muestral con estos datos fue de 208.

La muestra real obtenida fue de 254 personas, quienes fueron abordadas de forma directa mediante el instrumento desarrollado y alojado en la plataforma Qualtrics. Se estableció una restricción para que las preguntas de percepción sólo fueran contestadas por personas que tengan un smartphone y que hayan estado expuesta a publicidad en redes sociales. Los datos obtenidos fueron depurados respecto a valores perdidos y atípicos, quedando así una muestra final de 211.

Mediante el programa SPSS Statistics 25 se obtuvieron las correlaciones entre ítems, las que fueron significativas en su gran mayoría. La fiabilidad del instrumento se realizó mediante el Alfa de Cronbach (tabla 1), el cual es un estadístico que revela la consistencia del instrumento utilizado, en otras palabras, qué tan confiable es el conjunto de ítems usados para la medición (Hair, Black, Babin y Anderson, 2014). El Alfa de Cronbach obtenido fue de 0,957 para la variable PM, 0,929 para VMBC y 0,94 para CGU, datos que son superiores al mínimo aceptable de 0,7 (Nunnally, 1978).

Tabla 1. Alfa de Cronbach por dimensión y variable

\begin{tabular}{|c|c|c|c|}
\hline Variable & $\begin{array}{l}\text { Alfa de Cronbach } \\
\text { de Variable }\end{array}$ & Dimensión & $\begin{array}{l}\text { Alfa de Cronbach } \\
\text { por Dimensión }\end{array}$ \\
\hline \multirow{5}{*}{ PM } & \multirow{5}{*}{0,957} & Sinceridad (11 ítems) & 0,892 \\
\hline & & Emocionalidad (11 ítems) & 0,908 \\
\hline & & Competencia (9 ítems) & 0,872 \\
\hline & & Sofisticación (6 ítems) & 0,883 \\
\hline & & Rudeza (5 ítems) & 0,781 \\
\hline CGU & 0,94 & $\begin{array}{l}\text { Variable Unidimensional (4 } \\
\text { ítems) }\end{array}$ & 0,94 \\
\hline \multirow{3}{*}{ VMBC } & \multirow{3}{*}{0,92} & Lealtad de Marca (3 ítems) & 0,846 \\
\hline & & $\begin{array}{l}\text { Notoriedad/Asociaciones de } \\
\text { Marca (9 ítems) }\end{array}$ & 0,877 \\
\hline & & Calidad percibida (4 ítems) & 0,863 \\
\hline
\end{tabular}




\section{Resultados}

El $60 \%$ de la muestra fueron mujeres y el $40 \%$ hombres. En cuanto al uso de redes sociales, el 92\% usan Facebook, 75\% Instagram, un 40\% Twitter y solo $15 \%$ Snapchat. Es de mencionar que el $7 \%$ de los sujetos de la investigación respondieron que usan WhatsApp, la cual, aunque no es realmente una red social, sí la identifican como tal. También es remarcable que $44,1 \%$ usan smartphone gama premium, 23,7\% gama alta, $26,5 \%$ gama media y solo $5,7 \%$ poseen de gama baja.

Para realizar análisis factorial se verificó el cumplimiento del supuesto estadístico necesario de que existen correlaciones significativas entre todos los ítems. Esto se llevó a cabo mediante una matriz de correlaciones bivariadas, las que se mostraron en su gran mayoría significativas. Asimismo, se usó la matriz de correlaciones anti imagen, la cual es una forma de corroborar las correlaciones entre los ítems; y esta mostró para todos los casos datos superiores a 0,5 en la diagonal, el cual es el mínimo recomendado (FríasNavarro y Pascual Soler, 2012).

Después, mediante el software se obtuvieron los índices de esfericidad de Bartlett y Kaiser-Meyer-Olkin o KMO, los cuales son pruebas estadísticas más precisas y estrictas para determinar si las correlaciones entre ítems son significativas (Pérez y Medrano, 2010). Estos resultaron satisfactorios para todas las variables, pues el mínimo aceptado para KMO es 0,7 y la referencia para la esfericidad de Bartlett es una significancia de 0,05 o menor (FríasNavarro y Pascual Soler, 2012; Mejía Trejo, 2017). Esto se refleja en la tabla 2.

Tabla 2. Kaiser-Mayer Olkin y esfericidad de Bartlett para las variables

\begin{tabular}{|c|r|r|}
\hline Variable & KMO & $\begin{array}{c}\text { Significancia } \\
\text { esfericidad } \\
\text { de Bartlett }\end{array}$ \\
\hline PM & 0,932 & 0,000 \\
\hline CGU & 0,751 & 0,000 \\
\hline VMBC & 0,919 & 0,000 \\
\hline
\end{tabular}

Fuente: elaboración propia

El análisis factorial se hizo mediante el método de extracción de Componentes Principales y rotación de Máxima Verosimilitud (Varimax). Componentes Principales se le denomina a un método que permite estudiar las relaciones entre variables correlacionadas, transformando el conjunto de datos en grupos de variables que no están correlacionadas entre sí (Hair, Anderson, Tatham y Black, 1999). La rotación es un término que proviene de la representación gráfica geométrica de los datos y los componentes y que se 
realiza con el fin de obtener una agrupación de componentes más adecuada o una solución factorial que se aproxime lo más posible a lo que en estadística se denomina una estructura simple, es decir, que cada ítem tenga una correlación más próxima a 1 con uno de los componentes y cercana a 0 con el resto de componentes (Perez y Medrano, 2010).

Se obtuvieron tres componentes para VMBC, cinco para PM y uno para CGU, ya que esta es una variable unidimensional. Se obtuvo, además, que para la variable VMBC se explica el 69\% de la varianza, dato que para PM es del $65,4 \%$ y para CGU el $89,3 \%$, los cuales son aceptables dado que son superiores al mínimo del $60 \%$ para las Ciencias Sociales (Hair Jr., Black, Babin y Anderson, 2014).

Adicionalmente, se extrajo la validez convergente de los componentes obtenidos mediante la Varianza Media Extraída (AVE), que es una medición estadística de la correlación entre los ítems y las dimensiones de una variable, la cual, de manera similar a la Fiabilidad Compuesta (CR), permite saber si se está midiendo lo que se desea medir (Hair Jr. et al., 2014; Calvo-Porral, Martínez-Fernandez y Juanatey-Boga, 2013). Para las variables VMBC y PM, se obtuvieron valores de AVE y CR aceptables, es decir, superiores a 0,5 y a 0,8 respectivamente (Hair, Black, Babin y Anderson, 2014), exceptuando la dimensión rudeza de la PM, la que arrojó un AVE de 0,451, razón por la que se consideró eliminarla del modelo (Toldos-Romero y Orozco-Gómez, 2015). Esto es apreciable en la tabla 3.

Tabla 3. AVE y CR para cada dimensión

\begin{tabular}{|l|l|c|c|}
\hline Variable & \multicolumn{1}{|c|}{ Dimensión } & AVE & CR \\
\hline \multirow{4}{*}{ VMBC } & Lealtad de marca (LM) & 0,674 & 0,861 \\
\cline { 2 - 4 } & Notoriedad/asociaciones de marca (NAM) & 0,514 & 0,809 \\
\cline { 2 - 4 } & Calidad percibida (CP) & 0,626 & 0,869 \\
\hline \multirow{4}{*}{ PM } & Sinceridad (SIN) & 0,513 & 0,893 \\
\cline { 2 - 4 } & Emocionalidad (EMO) & 0,537 & 0,901 \\
\cline { 2 - 4 } & Competencia (COM) & 0,509 & 0,860 \\
\cline { 2 - 4 } & Sofisticación (SOFI) & 0,733 & 0,892 \\
\hline
\end{tabular}

Fuente: elaboración propia

Se llevó a cabo el análisis factorial confirmatorio en IBM AMOS 25, con el que se construyó y comprobó el modelo propuesto, así como la relación entre sus variables. La dimensión rudeza mostró un aporte bajo a la variable PM (inferior a 0,7) y, aunado al dato de AVE que fue menor a 0,5, se decidió eliminarla con el fin de procurar mejor robustez y parsimonia del modelo (Toldos-Romero y Orozco-Gómez, 2015; Geuens et al., 2009). 
Para conocer si el modelo es adecuado y si representa realmente los datos reales de la población, se llevan a cabo pruebas estadísticas llamadas indicadores de ajuste del modelo. Dentro de los indicadores de ajuste absoluto se obtuvo un CMIN/DF de 1,649, indicando un ajuste adecuado y por parte de los indicadores de ajuste comparativo, se obtuvo un CFI de 0,987, el TLI de 0,981 y NFI de 0,968 , los cuales fueron aceptables, ya que son mayores a 0,95 (Schermelleh-Engel, Moosbrugger y Müller, 2003). Respecto al ajuste parsimonioso, el PNFI resultó de 0,667. Por último, el RMSEA fue de 0,056, lo que, según el criterio de 0,08, indica un ajuste admisible (Rodríguez Ayán y Ruiz Díaz, 2008). En la tabla 4 se pueden apreciar un resumen de los indicadores de ajuste del modelo y en la figura 2 el modelo construido en el software.

Tabla 4. Resumen de indicadores de ajuste del modelo

\begin{tabular}{|l|c|l|}
\hline Indicador & Valor & $\begin{array}{c}\text { Criterio de aceptabilidad } \\
\text { (Schermelleh-Engel et al., 2003) }\end{array}$ \\
\hline CMIN/DF & 1,649 & Menor que 3 \\
\hline CFI & 0,987 & Mayor o igual a 0,95 \\
\hline TLI & 0,981 & Mayor o igual a 0,95 \\
\hline NFI & 0,968 & Mayor que 0,90 \\
\hline PNFI & 0,667 & Cercano a 1 \\
\hline RMSEA & 0,056 & Menor o igual a 0,08 \\
\hline
\end{tabular}

Fuente: elaboración propia

Figura 2. Modelo (IBM AMOS 25)

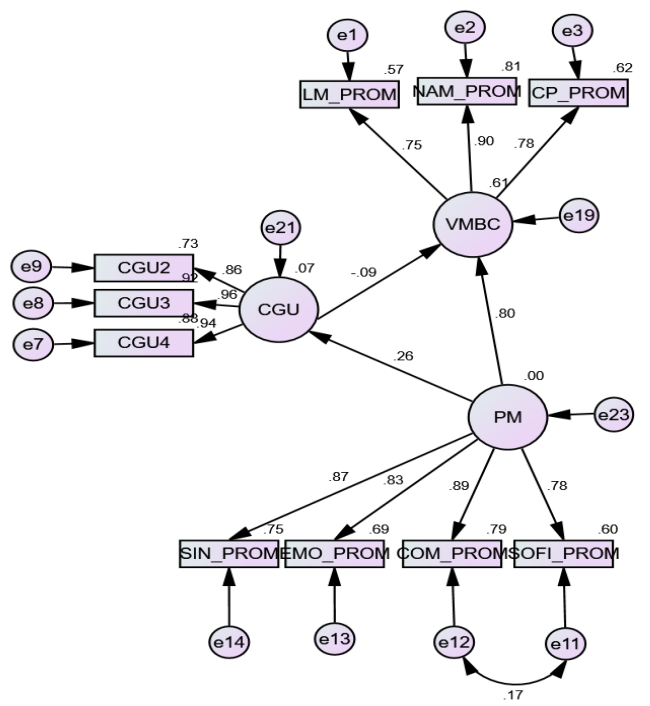

Fuente: elaboración propia 
En relación a las hipótesis planteadas, en la tabla 5 se pueden observar cada una de las hipótesis, la significancia de la prueba estadística y el resultado de aceptación o rechazo. La hipótesis de investigación es aceptada cuando el valor de la significancia es inferior a 0,05 y rechazada cuando supera a 0,05 (Hair et al., 1999).

Tabla 5. Resumen de pruebas de hipótesis de modelo

\begin{tabular}{|l|r|r|}
\hline Hipótesis & $\begin{array}{c}\text { Significancia } \\
\text { (Los valores cercanos a } \mathbf{0} \text { se } \\
\text { expresan con asteriscos) }\end{array}$ & $\begin{array}{r}\text { Aceptada }(<0.05) / \\
\text { Rechazada (>0.05) } \\
\text { (Hair et al., 1999) }\end{array}$ \\
\hline H1 & $* . * * *$ & Aceptada \\
\hline H2 & $* . * * *$ & Aceptada \\
\hline H3 & 0,066 & Rechazada \\
\hline \multicolumn{2}{|c|}{ Fuente: elaboración propia }
\end{tabular}

\section{Discusión}

Después de haberse realizado el tratamiento de datos mediante el análisis factorial, se ha encontrado que, tal como se planteó en las hipótesis de trabajo 1 , se comprueba que la personalidad de marca (PM) tiene un impacto directo y positivo sobre los contenidos generados por los usuarios (CGU), lo cual significa que, en efecto, cuanto más fuerte es la PM en la mente de los consumidores, estos generarán más comentarios acerca de la misma y los compartirán consecuentemente en las redes sociales. Dicho en otras palabras, los usuarios perciben que lo que transmite cada marca de smartphone en términos de su personalidad tiene influencia sobre la cantidad de contenidos que desean compartir acerca de la misma.

Lo anterior está en línea con lo referido por Dickinger y Lalicic (2015), no obstante contrasta con lo postulado por Walsh et al. (2013), quienes afirman que la relación es inversa, es decir, que son los CGU los que impactan en la personalidad de marca, en otras palabras que las comunicaciones que consumidores llevan a cabo acerca de una marca en las redes sociales influyen en la percepción de los demás acerca de los rasgos de personalidad que le adjudican. Empero, la presente investigación no descarta esta relación entre las variables y podría ser un aspecto a investigar en el futuro.

Aaker D.A. (1991) y Aaker J.L. (1997) han postulado que la personalidad de marca es un aspecto de relevancia para la generación de valor de marca (VMBC), mientras que Fournier (1998) la declara como base para la generación y mantenimiento de la relación que el consumidor tiene con las marcas y el valor que se les asigna. En línea con ellos, así como con Bouhlel, Mzoughi, Hadiji y Slimane (2011) y Vahdati y Mousavi Nejad (2016), mediante la comprobación 
de la segunda hipótesis (H2) se puede afirmar que la personalidad de marca es antecedente de la generación de valor para las marcas en las redes sociales, en otras palabras, que el valor que el consumidor percibe que obtiene de una marca va a ser interpretado como más fuerte en la medida que los rasgos de personalidad que le adjudique sean interpretados como congruentes con su propia personalidad humana (Toldos-Romero y Orozco-Gómez, 2015).

Este hallazgo ayuda a entender y confirmar que los consumidores crean relaciones con las marcas a través de la personalidad que le confieren a ellas. Estas relaciones nacen y se fomentan aún más cuando las marcas reafirman su propia personalidad, la cual se puede hacer expresa a través de distintos medios, entre los que se encuentran las redes sociales (Paschen et al., 2017; Yun et al., 2019).

A pesar de ello, estos contenidos que publican los usuarios en las redes sociales no necesariamente generan mayor valor de marca, al menos no para el caso de marcas de smartphones, según revela el presente estudio ya que, en contraste con lo postulado por Schivinski y Dabrowski (2015, 2016); Bruhn et al., (2012) y Bambauer-Sachse y Mangold (2011), se encontró que los contenidos que comparten los consumidores en redes sociales no muestran relación directa con la adjudicación de valor. Las razones exactas de esto podrían ser abordadas en una investigación posterior, no obstante, puede presumirse que hay aspectos culturales que influyen en ello.

Finalmente, dado que la tercera hipótesis (H3) no pudo ser comprobada, es posible afirmar que los contenidos que los usuarios comparten en redes sociales (CGU), no muestran influencia directa sobre el valor que los consumidores perciben de las marcas. Es decir, lo que ven, escuchan y leen los usuarios que sus pares comentan acerca de una marca no necesariamente impactará en la adjudicación de valor acerca de ella.

Sin embargo, con este hallazgo no podría concluirse que definitivamente los CGU no tienen impacto en absoluto sobre el VMBC, dado que la presente investigación está limitada a conocer si existe influencia directa, cuando esta podría darse de forma indirecta con la intervención de alguna otra variable como la actitud hacia la marca (Schivinski y Dabrowski, 2016). Posiblemente los consumidores de la ciudad de Guadalajara (México) no se basan únicamente en los comentarios de otros para asignar valor a las marcas de smartphones y existen otros elementos interventores que en conjunto conforman la percepción de valor.

En resumen, los resultados obtenidos con base en el modelo presentado, demuestran que la personalidad que los consumidores perciben de la marca 
influye en la generación de contenidos en las redes sociales, sin que estos impacten directamente en el valor percibido acerca de ésta. En contraste, la personalidad que los usuarios adjudican a la marca sí tiene influencia directa sobre cuánto valor confieren que obtienen de ella.

\section{Conclusiones}

En general, las investigaciones acerca de cómo el valor de marca con base en el consumidor (VMBC) es impactado por los contenidos generados por los usuarios (CGU) y por la personalidad de marca (PM) no son numerosas en el contexto de las redes sociales en México. Contrastando con estudios en otros contextos, a pesar que la personalidad de marca sí presenta relación directa con el VMBC y los CGU, estos últimos no muestran un impacto significativo en la creación de valor.

Este hallazgo supone un reto para los decisores en las organizaciones, ya que estas deben llevar a cabo acciones de mercadotecnia y comunicaciones de marca tanto online como offline que ayuden a incrementar a la percepción de rasgos de personalidad que sean acordes a los de sus públicos meta a fin de que el valor de marca sea mayor. Para que los consumidores le adjudiquen más valor es deseable que una marca sea percibida con diferentes rasgos de personalidad dependiendo del segmento al que se dirige, es decir, congruente con los rasgos de personalidad de cada tipo de consumidor.

Así, las marcas que son percibidas como más sinceras, por ejemplo, podrían ser mercadeadas y dirigidas a segmentos con tendencias a tener una personalidad más social o de tipo relacional. En esa misma línea, una marca que se comunique de manera sofisticada sería más conveniente posicionarla entre consumidores con personalidades que estén en concordancia con estos rasgos, ya que las marcas impactarán de manera distinta dependiendo de esta característica.

Asimismo, al momento de planificar acciones mercadológicas las organizaciones tienen que tomar en cuenta que el valor de una marca no emana directamente de la generación de contenidos que los consumidores provoquen en las redes sociales, pues es posible que en el contexto mexicano existan otras variables intervinientes en la comunicación digital. Además, el VMBC tiene distintas fuentes y no debe ser adjudicada nada más a los CGU, pues autores como Bruhn et al. (2012), Schivinski y Dabrowski (2016) plantean que los contenidos que las empresas crean y difunden en las redes sociales también presentan influencias sobre la percepción de valor.

Esta investigación propone un nuevo aporte cuantitativo que puede servir de base para los tomadores de decisión, pues con ella tienen un 
fundamento científico para sustentar propuestas o campañas en sus redes sociales encaminadas a generar mayor valor percibido de sus marcas por parte de los consumidores, lo cual es un precursor de la intención de adquirir el producto.

\section{Limitantes}

Para futuras investigaciones debe de considerarse que el ámbito de investigación del presente estuvo delimitado a la zona metropolitana de la ciudad de Guadalajara, por lo que se sugiere cautela al extrapolar los hallazgos presentados. En referencia al tamaño de la muestra, aunque este es estadísticamente válido, para otros estudios podría utilizarse uno mayor con el fin de proveer de más robustez a la información y disminuir el error estadístico.

Análogamente, es debido mencionar que se utilizó un solo tipo de producto (smartphones) para analizar la relación entre las variables, por lo que un aporte complementario sería contrastar los resultados de un estudio realizado con base en distintos tipos de productos tomando en cuenta factores de los mismos como el nivel de involucramiento o diferenciando aspectos demográficos de los consumidores, tales como su nivel socioeconómico.

Aunque este estudio presenta la interacción entre las variables CGU, PM y VMBC, autores como Schivinski y Dabrowski (2016), Faircloth, Capella y Alford (2015) y Lee y Kang (2013) postulan una relación indirecta mediada por otras variables adicionales como la Actitud hacia la Marca, la Imagen de Marca, la Relación con la Marca, entre otras no consideradas en esta investigación, por lo que, para futuros estudios, se podrían tomar estas en cuenta con el objeto de obtener una visión más amplia de cómo la percepción de marca, consumidores y organizaciones se interrelacionan entre sí.

Por último, dados los hallazgos contrastantes con otros autores sobre el impacto de los CGU sobre el VMBC, se abre una ventana a otras investigaciones que determinen si existen variables que intervengan en esta relación, así como para comprobar si existen factores propios de la cultura mexicana que provoquen que estas dos variables no se relacionen directamente como se observa en otros contextos.

\section{Referencias bibliográficas}

AAKER, D.A. (1991). Managing Brand Equity. Nueva York, Nueva York, EEUU:

The Free Press. https://www.inovaconsulting.com.br/wpcontent/uploads/2016/09/managing-brand-equity-by-davidaaker.pdf 
AAKER, J.L. (1997). Dimensions of brand personality. Journal of Marketing Research, 34(3), 347-356. doi.org/10.2139/ssrn.945432

AgGarWAL, P. y McGill, A. L. (2012). When Brands Seem Human, Do Humans Act Like Brands? Automatic Behavioral Priming. Effects of Brand Anthropomorphism. Journal of Consumer Research, 39(2), 307-323. doi.org/10.1086/662614

AKIN, M. (2011). Predicting Consumers' Behavioral Intentions with Perceptions of Brand Personality: A Study in Cell Phone Markets. International Journal of Business and Management, 6(6). doi.org/10.5539/ijbm.v6n6p193

Anderson, M.H., RAJU, S. y KoRDROSTAMI, M. (2018). Brand Personality and Organizational Pesonality: Do Consumers Differentiate Between Them? En D. Gal, K. Hewett, \& S. Jayachandran (ed.), AMA Summer Academic Conference, (págs. CB-48 - CB-52). Boston, Massachussetts.

https://www.researchgate.net/publication/324792510_Brand_Pers onality_and_Organizational_Personality_Do_Consumers_Differentiat e_Between_Them

Asociación de Internet. (2018). Estudios de la Asociación de Internet. Obtenido de Asociación de Internet: https://www.asociaciondeinternet.mx/es/estudios

BADII, M.H., CASTILLO, J. y GUILLEN, A. (2017). Tamaño óptimo de la muestra. Innovaciones de Negocios, 5(9). http://revistainnovaciones.uanl.mx/index.php/revin/article/view/199

BAMBAUER-SACHSE, S. y MANGOLD, S. (2011). Brand equity dilution through negative online word of mouth communication. Journal of Retailing and Consumer Services, 18, 38-45. doi.org/10.1016/j.jretconser.2010.09.003

Bouhlel, O., Mzoughi, N., HADIJI, D. y SLIMANE, I.B. (2011). Brand Personality's Influence on the Purchase Intention: A Mobile Marketing Case. International Journal of Business and Management, 6(9), 210-227. doi.org/10.5539/ijbm.v6n9p210

BRUHN, M., SCHOENMUELLER, V. y SCHAFER, D.B. (2012). Are social media replacing traditional media in terms of brand equity creation? Management Research Review, 35(9), 770-790. doi.org/10.1108/01409171211255948

BuIL, I., MARTíNEZ, E. y DE CHERNATONY, L. (2013). The influence of brand equity on consumer responses. Journal of Consumer Marketing, 30(1), 62-74. doi.org/10.1108/07363761311290849 
Calvo-Porral, C., Martínez-Fernandez, V.A. y JuAnatey-Boga, O. (2013).

Análisis de Dos Modelos de Ecuaciones Estructurales Alternativos para Medir la Intención de Compra. Revista Investigación Operacional, 34(3), 230-243.

https: / / www.researchgate.net/publication/257334461_Analisis_de _dos_modelos_de_ecuaciones_estructurales_alternativos_para_med ir_la_intencion_de_compra

CHEN, C.F. y PHOU, S. (2013). A closer look at destination: Image, personality, relationship and loyalty. Tourism Management(36), 269-278.

doi.org/10.1016/j.tourman.2012.11.015

Christodoulides, G., Jevons, C. y Bonhomme, J. (2012). Memo to Marketers: Quantitative Evidence for Change: How User-Generated Content Really Affects Brands. Journal of Advertising Research, 52(1), 1-13. doi.org/10.2501/jar-52-1-053-064

Data Reportal. (31 de Enero de 2019). Digital 2019: Global Digital Overview. Recuperado el 8 de Mayo de 2019, de Data Reportal:

https: / / datareportal.com/reports/digital-2019-global-digitaloverview

DELlarocas, C. (2003). The Digitalization of Word-Of-Mouth: Promises and Challenges of Online Feedback Mechanisms. Management Science, 49(10), 1407-1424. doi.org/10.2139/ssrn.393042

DiCKINGER, A. y LALICIC, L. (2015). «This City Is Absolutely Fun and Trendy»: A Destination Brand Personality Analysis in a Web 2.0 Setting. Information and Communication Technologies in Tourism, 321-333.

doi.org/10.1007/978-3-319-14343-9_24

Ding, Y., Phang, C.W., Lu, X., ChuAn-Hoo, T. y SuTANTO, J. (2014). The Role of Marketer- and User-generated Content in Sustaining the Growth. 2014 47th Hawaii International Conference on System Sciences (1.785-1.792). Hawaii: IEEE. doi.org/10.1109/hicss.2014.226

ESCOBAR-FARFÁN, M., SÁNCHEZ, C.M. y ARAYA-CASTILlO, L. (2016). Evolución y Descripción de los Modelos de personalidad de marca en Latinoamérica. Dimensión Empresarial, 14(2), 91-113. doi.org/10.15665/rde.v14i2.736 FAIRCLOTH, J.B., CAPELLA, L.M. y ALFORD, B.L. (2015). The Effect of Brand Attitude and Brand Image on Brand Equity. Journal of Marketing Theory and Practice, 9(3), 61-75. doi.org/10.1080/10696679.2001.11501897

FouRNIER, S. (1998). Consumers and Their Brands: Developing Relationship Theory in Consumer Research. The Journal of Consumer Research, 24(4), 343-373. doi.org/10.1086/209515 
FríAS-NAVARRo, D. y PAScual Soler, M. (2012). Prácticas del Análisis Factorial Exploratorio (AFE) en la Investigación sobre Conducta del Consumidor y Marketing. Suma Psicológica, 19(1), 47-58. doi.org/10.14349/sumapsi2012.1141

GENSLER, S., VolCKNER, F., LIU-ThompKins, Y. y WiERTZ, C. (2013). Managing Brands in the Social Media Environment. Journal of Interactive Marketing, 27(4), 242-256. doi.org/10.1016/j.intmar.2013.09.004

Geuens, M., WeijTERS, B. y DE Wulf, K. (2009). A New Measure of Brand Personality. International Journal of Research in Marketing, 26(2), 97-107. doi.org/10.1016/j.ijresmar.2008.12.002

GOLDBERG, L. R. (1990). An alternative description of personality: The big five factor structure. Journal of Personality and, 59(6), 1216-1229. doi.org/10.1037//0022-3514.59.6.1216

GonZÁLEZ HERnÁNDEZ, E.M., OROZCo GOMEZ, M.M. y BARRIOS, A.D. (2011). El valor de la marca desde la perspectiva del consumidor: Estudio empírico sobre la preferencia, lealtad y experiencia de marca en procesos de alto y bajo involucramiento de compra. Contaduría y Administración(235), 217-239. http://www.scielo.org.mx/scielo.php?script=sci_arttext\&pid=S0186 $-10422011000300011$

HAIR, J.F., ANDERSON, R.E., TATHAM, R.L. y BlACK, W.C. (1999). Análisis Multivariante (5a ed.). Madrid: Prentice Hall Iberia.

HAIR, J.F., BLACK, W.C., BABIN, B.J. y Anderson, R.E. (2014). Multivariate Data Analysis. Edimburgo: Pearson Education Limited. shorturl.at/IFOU3

KELLER, K.L. (1993). Conceptualizing, measuring and managing customerbased brand equity. Journal of Marketing, 57, 1-22. doi.org/10.1177/002224299305700101

KIM, C. K., HAN, D. y PARK, S.B. (2001). The effect of brand personality and brand identification on brand loyalty: Applying the Theory of Social Identification. Japanese Psychological Research, 195-206. doi.org/10.1111/1468-5884.00177

KIM, M. y SonG, D. (2017). When brand-related UGC induces effectiveness on social media: the role of content sponsorship and content type. International Journal of Advertising, 37(1), 105-124. doi.org/10.1080/02650487.2017.1349031

KudeshiA, C. y KumAR, A. (2017). Social eWOM: Does it affect the brand attitude and purchase intention of brands? Management Research Review, 40(3), 138. doi.org/10.1108/mrr-07-2015-0161 
LEE, H.J. y KANG, M.S. (2013). The Effect of Brand Personality on Brand Relationship, Attitude and Purchase Intention with a Focus on Brand Community. Academy of Marketing Studies Journal, 17(2), 85-97.

https: / / www.researchgate.net/publication/286498674_The_effect of_brand_personality_on_brand_relationship_attitude_and_purchas e_intention_with_a_focus_on_brand_community

LOUREIRO, S.M., LOPES, R. y KAUFMANN, H.R. (2014). How brand personality, brand identification and service quality influence service brand equity. Cogent Business y Management, 1(1). doi.org/10.1080/23311975.2014.981329

MAEHLE, N. y SHNEOR, R. (2009). On Congruence between Brand and Human Personalities. Journal of Product and Brand Management, 19(1), 44-53. doi.org/10.1108/10610421011018383

MEjÍA TREjo, J. (2017). Las Ciencias Administrativas y El Análisis Multivariante. Proyectos De Investigación, Análisis Y Discusión De Resultados. Técnicas Interdependientes (vol. 2). Zapopan, Jalisco, Mexico: Centro Universitario de Ciencias Económico Administrativas.

https: / / ssrn.com/abstract $=\mathbf{3 0 0 9 0 8 3}$

Nunnally, J. (1978). Psychometric Theory. Nueva York: McGraw-Hill. doi.org/10.2307/1161962

PAGE, M., FIRTH, C. y RAND, C. (2016). The Internet Value Chain. Londres: A.T. Kearney. Recuperado el 25 de julio de 2019: www.gsma.com/internetvalue-chain

PASCHEN, J., PITT, L., KiETZMAnN, J. y FARSHID, M. (2017). The brand personalities of brand communities: An analysis of online communication. Online Information Review. doi.org/10.1108/OIR-08-2016-0235

PEREZ, E.R. y MEDRANO, L. (2010). Análisis Factorial Exploratorio: Bases Conceptuales y Metodológicas. Revista Argentina de Ciencias del Comportamiento (RACC), 2(1), 58-66.

Rodgers, S. y Thorson, E. (2000). The Interactive Advertising Model: How Users Perceive and Process Online Ads. Journal of Interactive Advertising, 1(1), 42-61. doi.org/10.1080/15252019.2000.10722043

RoDríGUEZ AYÁN, M.N. y RuIZ DÍAZ, M.Á. (2008). Atenuación de la asimetría y de la curtosis de las puntuaciones observadas mediante transformaciones de variables. Psicológica, 29(2), 205-227.

https: / / dialnet.unirioja.es/servlet/articulo?codigo $=\mathbf{2 7 1 8 3 7 2}$

SASHI, C. (2012). Customer engagement, buyer-seller relationships, and social media. Management Decision, 50(2), 253-272.

doi.org/10.1108/00251741211203551 
SASMITA, J. y MoHD SUKI, M. (2015). Young consumers' insights on brand equity. International Journal of Retail y Distribution Management, 43(3), 276-292. doi.org/10.1108/ijrdm-02-2014-0024

SCHERMELLEH-EnGEL, K., MOOSBRUGGER, H. y MÜLLER, H. (2003). Evaluating the Fit of Structural Equation Models: Tests of Significance and Descriptive Goodness of Fit Meassures. Methods of Psychological Research Online, 8(2), 23-74.

http://citeseerx.ist.psu.edu/viewdoc/download?doi=10.1.1.509.425 8\&rep $=$ rep1\&type $=$ pdf

SCHIVINSKI, B. y DABROWSKI, D. (2015). The impact of brand communication on brand equity through Facebook. Journal of Research in Interactive Marketing, 9(1), 31-53. doi.org/10.1108/jrim-02-2014-0007

SCHIVINSKI, B. y DABROWSKI, D. (2016). The effect of social-media communication on consumer perceptions of brand. Journal of Marketing Communications, 22(2), 189-214. doi.org/10.1080/13527266.2013.871323

SCHIVINSKI, B., ŁUKASIK, P. y DRABROWSKI, D. (2015). User-generated images and its impact on consumer-based brand equity and on purchase intention. Logistyka, 2, 1.054-1.061.

http://irep.ntu.ac.uk/id/eprint/26432/1/PubSub3899_Schivinski.pdf

SCHOLZ, M., DORNER, V., LANDHERR, A. y PROBST, F. (2013). Awareness, Interest, and Purchase: The Effects of User and Marketer Generated Content on Purchase Decision Processes. Thirty Fourth International Conference on Information Systems, (1-17). Milán.

http://citeseerx.ist.psu.edu/viewdoc/download?doi=10.1.1.717.818 9\&rep=rep1\&type $=$ pdf

SMITH, A.N., FISCHER, E. y YoNGJIAN, C. (2012). How Does Brand-related Usergenerated Content Differ across YouTube, Facebook, and Twitter. Journal of Interactive Marketing, 102-113. doi.org/10.1016/j.intmar.2012.01.002

Socialbakers (s.f.). Free Social Media Statistics. Recuperado el 14 de Noviembre de 2016: https://www.socialbakers.com/statistics/

SU, J. y TONG, X. (2015). Brand personality and brand equity: evidence from the sportswear industry. Journal of Product y Brand Management, 24(2), 124133. doi.org/10.1108/JPBM-01-2014-0482

SU, J. y TONG, X. (2016). Brand Personality, Consumer Satisfaction, and Loyalty: A Perspective from Denim Jeans Brands. Family y Consumer Sciences Research Journal, 44(4), 427- 446. doi.org/10.1111/fcsr.12171

TOLDOS-ROMERO, M.d. y OROZCo-GómEZ, M.M. (2015). Brand personality and purchase intention. European Business Review, 27(5), 462-476. doi.org/10.1108/EBR-03-2013-0046 
VAHDATI, H. y MouSAVI NEJAD, S.H. (2016). Brand Personality Toward Customer Purchase Intention: The Intermediate Role of Electronic Word-Of-Mouth and Brand Equity. Asian Academy of Management Journal, 21(2), 1-26. doi.org/10.21315/aamj2016.21.2.1

VALETTE-FloRenCE, P., GuizANI, H. y MERUNKA, D. (2011). The impact of brand personality and sales promotions on brand equity. Journal of Business Research, 24-28. doi.org/10.1016/j.jbusres.2009.09.015

VICKERY, G. y WUNSCH-VINCENT, S. (2007). Participative Web and User-created Content: Web 2.0 Wikis and Social Networking. París: Organización para la Cooperación y Desarrollo Económico (OCDE). https://dl.acm.org/doi/book/10.5555/1554640

WALSH, P., CLAVIO, G., Lovell, M.D. y BLASZKA, M. (2013). Differences in Event Brand Personality Between Social Media Users and Non-users. Sport Marketing Quarterly, 22(4), 214-223.

http://www.academia.edu/download/34616439/differences_in_eve nt_brand_personality_between_social_media_users_and_nonusers.pdf

XIA, L. y BECHWATI, N.N. (2010). Word of Mouse: The Role of Cognitive Personalization in Online Consumer Reviews. Journal of Interactive Advertising, 9(1), 3-13. doi.org/10.1080/15252019.2008.10722143

Yoo, K.-H. y GRETZEL, U. (2011). Influence of personality on travel-related consumer-generated media creation. Computers in Human Behavior, 27, 609-621. doi.org/10.1016/j.chb.2010.05.002

Yun, J.T., PAMUKSUZ, U. y DuFF, B.R. (2019). Are we who we follow? Computationally Analyzing Human Personality and Brand Following on Twitter. International Journal of Advertising, 1-20.

doi.org/10.1080/02650487.2019.1575106 